\title{
Chaotic flows in microchannels: A lattice Boltzmann study
}

\author{
F. Varnik, D. Raabe \\ Max-Planck Institut für Eisenforschung \\ Max-Planck Straße 1, 40237 Düsseldorf, Germany
}

\begin{abstract}
Roughness effects on lubricant flows are investigated via 2D lattice Boltzmann simulations. At a Reynolds numbers of order 1000 a transition from laminar to unsteady flow is observed by an increase of the roughness height from about $10 \%$ to about $25 \%$ of the channel width. At lower Reynolds numbers (where the flow is laminar in both channels), the transition is observed when increasing the wall roughness further. In other words, the critical Reynolds number for the transition from laminar toward unsteady flow decreases at higher wall roughness. Wall roughness may, therefore, qualitatively change the flow properties in confined geometry. Due to the ubiquitous presence of the wall roughness, the phenomenon is relevant in all cases where relatively high Reynolds number flow occur in strongly confined channels such as lubricant flow during the deformation of solid surfaces. For a fixed Reynolds number and channel geometry, time and spatial dependence of the velocity field and fluctuating quantities obey the scaling behavior as expected from the structure of the NavierStokes (NS) equations. This underlines the physical significance of the observed transition. As a possible application, wall roughness may, therefore, be used in order to enhance mixing efficiency at a given Reynolds number.
\end{abstract}

Keywords: lattice Boltzmann method; chaotic mixing; flow at rough surfaces; flow instability

\section{Introduction}

Fluid mechanics at deformable metallic surfaces has long been studied within the framework of classical continuum-based metal/fluid tribology theory and macroscopic engineering experimentation in the context of sheet metal forming [1]. Since the last decade, more attention has been paid to the study fluid mechanics at deformable metallic surfaces also at the molecular dynamics scale [1].

Both methods of tackling fluid mechanics at deformable metallic surfaces are characterized by several limitations. The first set of limitations associated with conventional macroscopic continuum-based approaches along these lines lies in the scale. Classical works about tribology at metal/fluid interfaces which take a continuum perspective study fluid dynamics at the macroscopic or respectively statistical scale [1].

This means that fluid flow is usually assumed to be laminar and the metallic surface is anticipated to be perfectly flat. Both assumptions must be modified when it comes to a detailed analysis. Firstly, we recall that fluid velocities of order $100 \mathrm{~m} / \mathrm{s}$ ( $7 \%$ of the sound speed in water) or higher are quite common in rapid quenching processes [2]. Taking a channel of width $100 \mu \mathrm{m}$, and a dynamic viscosity of $\nu=10^{-5}$ (oil) yields Reynolds numbers of order Re $\sim 1000$. On the other hand, metallic surfaces are never flat but reveal an average roughness ranging from some nanometers to some tens of micrometers depending on the mechanical and microstructural boundary conditions. This applies in particular to the evolution of surface roughness during elastic-plastic deformation.

It is the purpose of the present report to show that, when these two facts (relatively high Reynolds number and non-negligible wall roughness) are combined together, non-trivial flow behavior may occur. It must be emphasized that we do not consider turbulent flows [3]. Rather, we wish to focus on situations where the flow is laminar and steady for channels with smooth and weakly rough walls but becomes unsteady (and progressively chaotic) as the wall roughness is increased. Reynolds numbers considered here are, therefore, far below the turbulent regime. They are, however, high enough so that Stokes approximation (neglecting the non-linear term in the NS-equation) does not hold.

\section{Simulation method}

The lattice Boltzmann method (LBM), a kinetic approach to solve the NS-equation is used. While direct derivations of the method now exist, the LBM has been historically devised as an improvement of the lattice gas cellular automata (LGCA) [4]-[9] providing e.g. a far better statistical accuracy [9]-[11]. In the past 20 years, the LBM has been extensively used as an efficient tool for the study of a variety of fluid flow problems such as two-phase flow through porous media [12], particlefluid suspensions [13], [14] and high Reynolds number turbulent flows [15]. 
There are excellent monographs [16]-[18] and comprehensive review articles [19], [13], [20] on the lattice Boltzmann method and the historically related lattice gas cellular automata (LGCA). Here, we give a short introduction of the method. A simplified view of the lattice Boltzmann method may be presented as follows: A fluid portion residing at a given point, $\boldsymbol{x}$, in space is divided into a small number of parcels, $f_{i}$, each moving with a well defined velocity, $\boldsymbol{c}_{i}$, on a lattice (see Fig. 1). During the time step $t \rightarrow t+1$ (note that $\Delta t=1)$, the fluid parcel, $f_{i}(\boldsymbol{x}, t)$, is first relaxed to its local equilibrium,-io $f_{i}^{\text {eq }}(\boldsymbol{x}, t)$, with a rate of $\omega$,

$$
f_{i}^{\prime}(\boldsymbol{x}, t)=f_{i}(\boldsymbol{x}, t)-\omega\left(f_{i}(\boldsymbol{x}, t)-f_{i}^{\mathrm{eq}}(\boldsymbol{x}, t)\right),
$$

and then freely propagated to the site $\boldsymbol{x}+\boldsymbol{c}_{i}$

$$
f_{i}\left(\boldsymbol{x}+\boldsymbol{c}_{i}, t+1\right)=f_{i}^{\prime}(\boldsymbol{x}, t) .
$$

Here, the post-collision population, $f_{i}^{\prime}$, is introduced to underline the formal separation of the relaxation and propagation steps. The relaxation rate is closely related to the fluid dynamic viscosity via

$$
\nu=\frac{1}{6}\left(\frac{2}{\omega}-1\right)
$$

The local equilibrium distribution, $f_{i}^{\text {eq }}$, is usually taken as a second order expansion of the Maxwell velocity distribution leading to

$$
f_{i}^{\mathrm{eq}}=\rho w_{i}\left[1+\frac{1}{c_{s}^{2}} \boldsymbol{u} \cdot \boldsymbol{c}_{i}+\frac{1}{2 c_{s}^{4}}\left[\left(\boldsymbol{u} \cdot \boldsymbol{c}_{i}\right)^{2}-c_{s}^{2} \boldsymbol{u} \cdot \boldsymbol{u}\right]\right],
$$

where $c_{s}$ is the sound speed and $w_{i}$ is a set of weights normalized to unity. For the two dimensional nine velocity model (D2Q9) used in our studies (see Fig. 1 for an illustration) one finds $w_{0}=4 / 9, w_{1}=w_{2}=w_{3}=$ $w_{4}=1 / 9$ and $w_{5}=w_{6}=w_{7}=w_{8}=1 / 36$ (see [18] for a short but comprehensive derivation). Once the discrete populations, $f_{i}$, are known, fluid density, $\rho(\boldsymbol{x}, t)$, and velocity, $\boldsymbol{u}(\boldsymbol{x}, t)$, at a given point and time are obtained via

$$
\rho=\sum_{i} f_{i}(\boldsymbol{x}, t) \quad \text { and } \quad \rho \boldsymbol{u}=\sum_{i} f_{i}(\boldsymbol{x}, t) \boldsymbol{c}_{i} .
$$

For the fluid-solid interaction, the bounce-back rule is used: Fluid populations arriving at a solid node are sent back to the node where they came from. At not too high viscosities, this leads to zero streaming velocity half-way between a solid node and its neighboring fluid node (stick boundary condition). In the following, all quantities are expressed in lattice Boltzmann (LB) units. The unit of length is the internode spacing, $\Delta x \equiv 1$. The time in measured in units of an iteration step, $\Delta t \equiv 1$. This fixes the unit of the velocity $\Delta x / \Delta t \equiv 1$. Comparison with real experiments is easily done by e.g. fixing the dynamic viscosity, $\nu$, and the sound speed, $c_{\mathrm{S}}$.
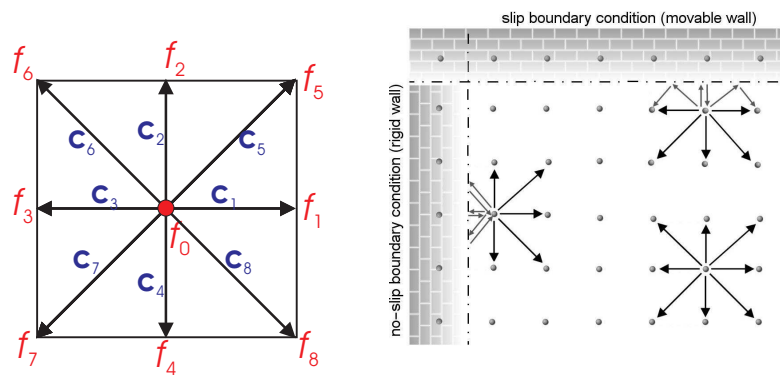

Figure 1: Left: Schematic view of two dimensional nine velocity (D2Q9) lattice Boltzmann Model. During the free propagation step, the population $f_{i}$ is transported along the velocity vector $\boldsymbol{c}_{i}$. The zero-velocity population, $f_{0}$, ensures correct hydrodynamic behavior in the compressible regime, i.e. at high Mach numbers (fluid velocity/sound speed). Right: Illustration of rules in order to realize stick or slip boundary conditions

\section{Results}

Figure 2 illustrates how a variation of the surface roughness alone may trigger transition toward unsteady flow. In the right panel of the figure, velocity versus time is shown for two choices of the wall roughness as described in the left panel. Here, horizontal, $U_{x}$ and vertical, $U_{y}$, components of the fluid velocity are monitored at a fixed point in the middle of the flow region. The time axis is rescaled by the momentum diffusion time $t_{\mathrm{d}}=H^{2} /(8 \nu)(H=$ effective channel width $)$ as can be estimated from a study of the Stokes flow in the same channel with parallel walls.

The fluid is at rest for $t<0$. At $t=0$ an external body force is switched on, which accelerates the fluid gradually toward a steady state flow ${ }^{1}$. In the channel with a 2:20 zig-zag surface, the fluid velocity in the direction parallel to the wall, $U_{x}$, increases continuously and smoothly until it reaches a constant value after a time of order $t_{\mathrm{d}}$ while the vertical component of the velocity, $U_{y}$, remains zero for all times.

A qualitative change in the flow behavior is, however, observed as the roughness height is increased from 2 to 5 lattice units (corresponding to a change of the roughness slope from 0.1 to 0.25 at the same roughness wave length). Now, both $U_{x}$ and $U_{y}$ exhibit strong fluctuations suggesting the presence of flow instability. A further hint on flow instability is the observed drop of the mean velocity at the onset of fluctuations, indicative of a higher rate of energy loss (and thus a higher friction force) due to the chaotic nature of the flow.

It must be emphasized here that the roughness-induced flow instability discussed above is not restricted to the specific choice of triangular obstacles. We do observe

\footnotetext{
${ }^{1}$ Note that we apply the term "steady state" not only for a time independent flow, but also for a time dependent flow, whose statistical properties do not change with time.
} 

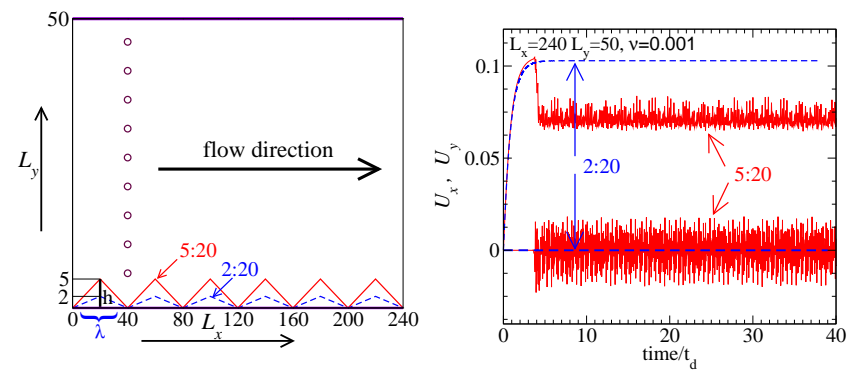

Figure 2: Left: A sketch of the simulation box for two choices of the wall roughness. The top wall is assumed to be flat, whereas the bottom wall has a zig-zag form. A zig-zag wall can be characterized by two numbers: The height, $h$, of the roughness tip and the half-wave length $\lambda / 2$. We use the notation $h: \lambda / 2$ for a given zig-zag wall. Flow velocity versus time is recorded at equidistant points along the line $x=40$ (open circles). Note that the region below the zig-zag line is filled with solid particles (not shown for clarity). Right: Parallel, $U_{x}$, and the perpendicular, $U_{y}$, components of the fluid velocity in the middle of the flow region versus time for both choices of the wall roughness shown in the left panel. The time axis is rescaled by the momentum diffusion time. Note the strong fluctuations of the vertical component of the velocity in the case of the 5:20 zig-zag surface.

the same phenomenon also for a variety of other regular surfaces as well as for a random distribution of roughness elements.

In order to examine the physical significance of the observed transition we have computed various quantities for which exact behavior can be derived from the NavierStokes equation. An example is the sum of the viscous stress $\nu \rho \partial<U_{x}>/ \partial y$ and the so-called Reynolds stress $-\rho<\delta U_{x} \delta U_{y}>$ which obeys a straight line with an slope, $\rho g,(g=$ imposed external force per unit mass $)$ : $\nu \rho<\frac{\partial U_{x}}{\partial y}>-\rho<u_{x} u_{y}>=\tau_{\mathrm{w}}+\rho g\left(y-y_{\mathrm{w}}\right)\left(y_{\mathrm{w}}=\right.$ the position of the wall, $\tau_{\mathrm{w}}=$ viscous stress at the wall). Indeed, this equation is very well satisfied by our simulation results [2].

A further, non-trivial, hint regarding the reliability of simulated results is to verify whether the solutions obtained within our simulations obey scaling rules as expected from the structure of the NS-equations: For a given Reynolds number and channel geometry the solution of the NS-equation is unique if expressed in appropriate dimensionless units. As shown in Figs.3 and 4 , the solutions obtained within our simulations satisfy this important reqirement.

Next we show in Fig. 5 how a proper channel design may dramatically improve the efficiency of mixing. As seen from this figure, the unstable character of the flow is enhanced by the removal of obstacles. In other words
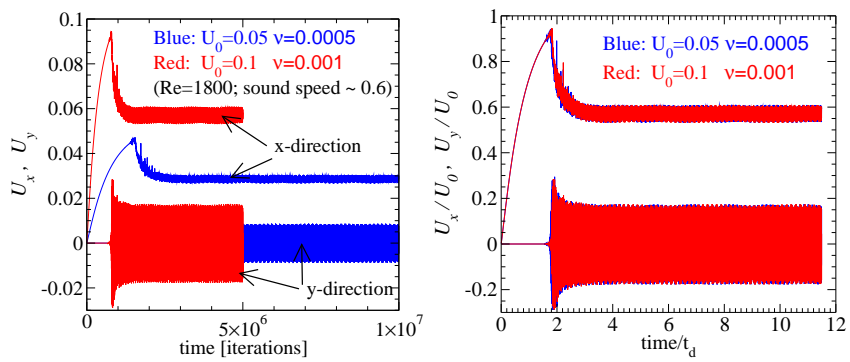

Figure 3: Scaling behavior of the velocity field. Left: Horizontal, $U_{x}$, and vertical, $U_{y}$, components of the streaming velocity are shown versus time for two choices of the parameter $U_{0}$ (the external force is set such that, in the case of a laminar flow, the mid-channel velocity would equal $U_{0}$ ). Left: Same as in the left panel, but rescaled. $U_{x}$ and $U_{y}$ are divided by the characteristic flow velocity, $U_{0}$, and the time is given in units of the momentum diffusion time, $t_{\mathrm{d}}$.

a dense array of obstacles may even stabilize the flow.

\section{Summary}

Results of lattice Boltzmann simulations on roughness-induced transition toward an unsteady flow are presented. The work is motivated by the fact that relatively high Reynolds number flows may occur in narrow channels in a variety of situations. The term "relatively high", here, means that we consider situations where the non linear term in the NS equation is no longer negligible, but the flow remains laminar and stable with respect to small perturbations. This brings about the relevance of the wall-roughness as an independent parameter affecting the flow properties: Wall roughness may serve as a source of large perturbations in the sense that these perturbations will not decay fast enough along the flow so that their advection with the flow can give rise to a complex and unsteady behavior.

In cases where the average roughness height is of order of $10 \%$ of the channel width or larger, we do indeed observe a crucial dependence of the flow on the wall roughness for Reynolds numbers of order 1000. The onset of unsteady flow is shifted toward lower Reynolds numbers as the roughness height is increased.

The physical significance of the obtained results is underlined by demonstrating that solutions obtained within the present simulations satisfy the scaling requirement of the NS equation: When all quantities such as velocity and the Reynolds stress are expressed in appropriate units, the solution is unique for all choices of the mean streaming velocity, system size etc. provided that the Reynolds number and the channel shape are unaltered.

The phenomenon reported here is of particular importance in all cases where the roughness height is of the 

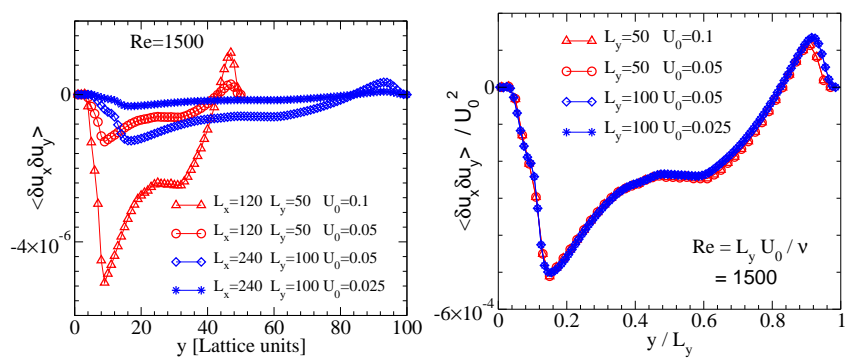

Figure 4: Scaling property of the Reynolds stress, $\tau_{\text {rey }}=<\delta U_{x} \delta U_{y}>(\delta U=U-<U>) . \quad \tau_{\text {rey }}$ is computer for different channel sizes and velocities as indicated in the figure while both the channel shape and the Reynolds number were kept unchanged. Right panel shows the same quantities now rescaled appropriately.
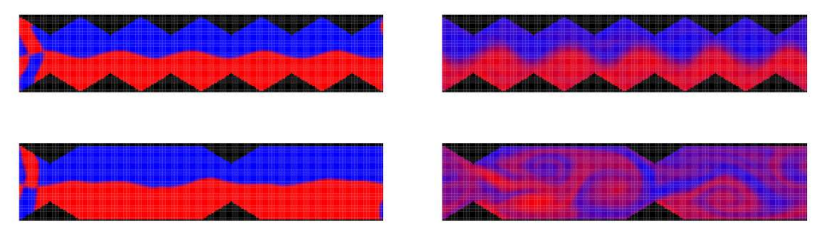

Figure 5: Impact of the channel design on mixing. In a zig-zag channel (top), 2/3 of all teeth are removed (bottom). A flow which is not that irregular (top) then becomes fully chaotic (bottom) leading to an efficient mixing. Left: initial stage. Right: after a time of $10^{-2} \times$ $t_{\text {diff }}$, where $t_{\text {diff }}$ is the time necessary for diffusion to achieve complete mixing.

order of $10 \%$ the channel width or higher. An example of considerable importance is lubricant flow occurring during the deformation of solid surfaces.

Finally, we also give an example how an appropriate design of the channel geometry may considerably enhance the chaotic nature of the flow (Fig. 5). In view of increasing number of potential applications of chaotic flows [21] in civil engineering, environmental industry (e.g. solution recovery) as well as in the medical science (e.g. enhanced chaotic mixing in microchannels), the results of our studies may find a wide range of applications as they open an alternative way for tuning flow properties.

Acknowledgments: $\mathrm{FV}$ is supported by the DFG under the grants Do794/1-1 and Va205/3-2.

\section{REFERENCES}

[1] N. Bay and T. Wanheim, Contact Phenomena under Bulk Plastic Deformation Conditions, Advanced Technology of Plasticity 4, 1677 (1990).

[2] F. Varnik and D. Raabe, Scaling effects in microscale fluid flows at rough solid surfaces, Modelling Simul. Mater. Sci. Eng. 14, 857 (2006)
[3] S. Pope, Turbulent Flows (Cambridge university press, Cambridge, UK, 2000); J. Mathieu and J. Scott, An Introduction to Turbulent Flow (Cambridge university press, Cambridge, UK, 2000).

[4] R. Benzi, S. Succi, and M. Vergassola, The LatticeBoltzmann Equation - Theory and Applications, Phys. Rep. 3, 145 (1992).

[5] L. Bunimovich, A. Lambert, and R. Lina, The Emergence of Coherent Structures in Coupled Map Lattices, J. of Stat. Phys. (1990).

[6] S. Chen, X. Shan, Z. Wang, and G. Doolen, Lattice-Boltzmann Computational Fluid Dynamics in Three Dimensions, J. of Stat. Phys. 68, 379 (1992).

[7] U. Frisch, B. Hasslacher, and Y. Pomeau, Lattice Gas Hydrodynamics in Two and Three Dimensions, Phys. Rev. Lett. 56, 1505 (1986).

[8] F. Higuera and J. Jimenez, Boltzmann Approach to Lattice-Gas Simulations, Europhysics letters 9, 663 (1989).

[9] G. McNamara and G. Zanetti, Use of the Boltzmann Equation to Simulate Lattice-Gas Automata, Phys. Rev. Lett. 61, 2332 (1988).

[10] F. Higuera, S. Succi, and R. Benzi, Lattice gas dynamics with enhanced collisions, Europhys. Lett. 9, 345 (1989).

[11] Y. Qian, D. d'Humieres, and P. Lallemand, Lattice BGK models for Navier-Stokes equation, Europhys. Lett. 17, 479 (1992).

[12] A. Gunstensen and D. Rothman, LatticeBoltzmann studies of two-phase flow through porous media, J. Geophys. Res. 98, 6431 (1993).

[13] A. Ladd and R. Verberg, Lattice-Boltzmann Simulations of Particle-Fluid Suspensions, J. Stat. Phys. 104, 1191 (2001).

[14] P. Ahlrichs and B. Dünweg, Simulation of a single polymer chain in solution by combining lattice Boltzmann with molecular dynamics, J. Chem. Phys. 111, 8225 (1999).

[15] S. Succi, O. Filippova, H. Chen, and S. Orszag, Towards a Renormalized Lattice Boltzmann Equation for Fluid Turbulence, J. Stat. Phys. 107, 261 (2002).

[16] S. Succi, The Lattice Boltzmann Equation: for Fluid Dynamics and Beyond (Series Numerical Mathematics and Scientific Computation) (Oxford University Press, Oxford, 2001).

[17] D. H. Rothman and S. Zaleski, Lattice-Gas Cellular Automata (Simple Models of Complex Hydrodynamics) (Cambridge University Press, Cambridge, 1997).

[18] D. Wolf-Gladrow, Lattice-Gas Cellular Automata and Lattice Boltzmann Models (Springer, Berlin Heidelberg, 2000).

[19] S. Chen and G. Doolen, Lattice Boltzmann method for fluid flows, Ann. Rev. Fluid Mech. 30, 329 
(1998).

[20] D. Raabe, Overview of the lattice Boltzmann method for nano- and microscale fluid dynamics in materials science and engineering, Modelling Simul. Mater. Sci. Eng. 12, R13 (2004).

[21] C. Simonnet and A. Groisman, Chaotic Mixing in a Steady Flow in a Microchannel, Phys. Rev. Lett. 94, 134501 (2005); A. D. Strook, S. K. W. Dertinger, A. Ajdari, I. Mezic, H. A. Stone, G. M. Whitesides, Chaotic Mixer for Microchannels, Science, 295, 647 (2002); M. C. Jullien, P. Castiglione and P. Tabeling, Experimental Observation of Batchelor Dispersion of Passive Tracers, Phys. Rev. Lett. 85, 3636 (2000); R. A. Truesdell, P. V. Vorobieff, L. A. Sklar and A. A. Mammoli, Mixing of a continuous flow of two fluids due to unsteady flow, Phys. Rev. E 67066304 (2003). 